\title{
A Scalarization Proximal Point Method for Quasiconvex Multiobjective Minimization *
}

\author{
H. C. F. Apolinario ${ }^{\dagger}$ \\ E. A. Papa Quiroz \\ P. R. Oliveira ${ }^{\S}$
}

October 15, 2018

\begin{abstract}
In this paper we propose a scalarization proximal point method to solve multiobjective unconstrained minimization problems with locally Lipschitz and quasiconvex vector functions. We prove, under natural assumptions, that the sequence generated by the method is well defined and converges globally to a Pareto-Clarke critical point. Our method may be seen as an extension, for the non convex case, of the inexact proximal method for multiobjective convex minimization problems studied by Bonnel et al. (SIAM Journal on Optimization 15, 4, 953-970, 2005).
\end{abstract}

Keywords: Multiobjective minimization, Clarke subdifferential, quasiconvex functions, proximal point methods, Fejér convergence, Pareto-Clarke critical point.

\section{Introduction}

In this work we consider the unconstrained multiobjective minimization problem:

$$
\min \left\{F(x): x \in \mathbb{R}^{n}\right\}
$$

where $F: \mathbb{R}^{n} \longrightarrow \mathbb{R}^{m}$ is a locally Lipschitz and quasiconvex vector function on the Euclidean space $\mathbb{R}^{n}$. A motivation to study this problem are the consumer demand theory in economy, where the quasiconvexity of the objective vector function is a natural condition associated to diversification of the consumption, see Mas Colell et al. [21], and the quasiconvex optimization models in location Theory, see [12]. Another motivation are the extensions of well known methods in convex optimization to quasiconvex one, we mentioned the following works:

\footnotetext{
${ }^{*}$ This research was conducted with partial financial support from CAPES, through the Interagency Doctoral Program New Frontiers UFRJ/UFT.

${ }^{\dagger}$ Federal University of Tocantins, Undergraduate Computation Sciences Course, ALC NO 14 (109 Norte) AV.NS.15 S/N , CEP 77001-090, Tel: +55 63 8481-5168; +55 63 3232-8027; FAX +55 63 3232-8020, Palmas, Brazil (hellenato@cos.ufrj.br).

${ }^{\ddagger}$ Mayor de San Marcos National University, Department of Ciencias Matemáticas, Lima, Perú. Federal University of Rio de Janeiro, Computing and Systems Engineering Department, post office box 68511,CEP 21945-970, Rio de Janeiro, Brazil(erikpapa@gmail.com). The research of the second author was supported by the Postdoctoral Scholarship CAPES-FAPERJ Edital PAPD-2011

${ }^{\S}$ Federal University of Rio de Janeiro, Computing and Systems Engineering Department, post office box 68511,CEP 21945-970, Rio de Janeiro, Brazil(poliveir@cos.ufrj.br).
} 
- Bello Cruz et al. [3, considered the projected gradient method for solving the problem of finding a Pareto optimum of a quasiconvex multiobjective function. They proved the convergence of the sequence generated by the algorithm to a stationary point and when the components of the multiobjective function are pseudoconvex, they obtained the convergence to a weak Pareto solution.

- da Cruz Neto et al. [10, extended the classical subgradient method for real-valued mi-nimization to multiobjective optimization. Assuming the basically componentwise quasiconvexity of the objective components they obtained the full convergence of the sequence to a Pareto solution.

- Papa Quiroz and Oliveira [24, 25, 27, have been extended the convergence of the proximal point method for quasiconvex minimization problems on general riemannian manifolds wich includes the euclidean space. Furthermore, in [26] the authors extended the convergence of the proximal point method for the nonnegative orthant.

- Kiwiel [16], extended the convergence of the subgradient method to solve quasiconvex minimization problems in Hilbert spaces.

- Brito et al. [6], proposed an interior proximal algorithm inspired by the logarithmicquadratic proximal method for linearly constrained quasiconvex minimization problems. For that method, they proved the global convergence when the proximal parameters go to zero. The latter assumption could be dropped when the function is assumed to be pseudoconvex.

- Langenberg and Tichatschke [17] studied the proximal method when the objective function is quasiconvex and the problem is constrained to an arbitrary closed convex set and the regularization is a Bregman distance. Assuming that the function is locally Lipschitz and using the Clarke subdifferential, the authors proved the global convergence of the method to a critical point.

In this paper we are interested in extending the convergence properties of the proximal point method to solve the quasiconvex multiobjective problem (11). The proximal point method, introduced by Martinet [20, to solve the problem $\min \left\{f(x): x \in \mathbb{R}^{n}\right\}$ where $f$ is a escalar function, generates a sequence $\left\{x^{k}\right\}_{k \in \mathbb{N}} \subset \mathbb{R}^{n}$, from an iterative process starting with a point $x^{0} \in \mathbb{R}^{n}$, arbitrary, and $x^{k+1} \in \operatorname{argmin}\left\{f(x)+\frac{\lambda_{k}}{2}\left\|x-x^{k}\right\|^{2}: x \in \mathbb{R}^{n}\right\}$, where $\lambda_{k}>0$, is a regularization parameter. It is well known, see Guler [13], that if $f$ is convex and $\left\{\lambda_{k}\right\}$ satisfies $\sum_{k=1}^{+\infty}\left(1 / \lambda_{k}\right)=+\infty$, then $\lim _{k \rightarrow \infty} f\left(x^{k}\right)=\inf \left\{f(x): x \in \mathbb{R}^{n}\right\}$. Furthermore, if the optimal set is nonempty, we obtain that $\left\{x^{k}\right\}$ converges to an optimal solution of the problem.

When $F$ is convex in (1), Bonnel at al. [5] have been proved the convergence of the proximal point method for a weak Pareto solution of the problem (11) in a general context, see also Villacorta and Oliveira [31] using proximal distances and Gregório and Oliveira [11] using a logarithmic quadratic proximal scalarization method.

In this work we introduce a scalarization proximal point method to solve the quasiconvex multiobjective minimization problem (1). The iteration is the following: given $x^{k} \in \mathbb{R}^{n}$, find 
$x^{k+1} \in \Omega_{k}$ such that:

$$
0 \in \partial^{o}\left(\left\langle F(.), z_{k}\right\rangle+\frac{\alpha_{k}}{2}\left\langle e_{k}, z_{k}\right\rangle\left\|\cdot-x^{k}\right\|^{2}\right)\left(x^{k+1}\right)+\mathcal{N}_{\Omega_{k}}\left(x^{k+1}\right)
$$

where $\partial^{o}$ is the Clarke subdifferential, $\Omega_{k}=\left\{x \in \mathbb{R}^{n}: F(x) \preceq F\left(x^{k}\right)\right\}, \alpha_{k}>0,\left\{e_{k}\right\} \subset \mathbb{R}_{++}^{m}$, $\left\|e_{k}\right\|=1,\left\{z_{k}\right\} \subset \mathbb{R}_{+}^{m} \backslash\{0\},\left\|z_{k}\right\|=1$ and $\mathcal{N}_{\Omega_{k}}\left(x^{k+1}\right)$ the normal cone to $\Omega_{k}$ at $x^{k+1}$.

We prove the well definition of the sequence generated by the method and we obtain the global convergence to a Pareto-Clarke critical point and when $F$ is convex we obtain the convergence to a weak Pareto solution of the problem.

The paper is organized as follows: In Section 2 we recall some concepts and results basic on multiobjective optimization, quasiconvex and convex functions, Fréchet, Limiting and Clarke subdiferential, descent direction and Fejér convergence theory. In Section 3 we introduce our method and analyze the convergence of the iterations. In Section 4 , we present some quasiconvex optimization models and in Section 5 we give our conclusion and some ideas for future researchers.

\section{Preliminaries}

In this section, we present some basic concepts and results that are of fundamental importance for the development of our work. These facts can be found, for example, in Hadjisavvas [14, Mordukhovich [23] and, Rockafellar and Wets [29].

\subsection{Definitions, notations and some basic results}

Along this paper $\mathbb{R}^{n}$ denotes an euclidean space, that is, a real vectorial space with the canonical inner product $\langle x, y\rangle=\sum_{i=1}^{n} x_{i} y_{i}$ and the norm given by $\|x\|=\sqrt{\langle x, x\rangle}$.

Given a function $f: \mathbb{R}^{n} \longrightarrow \mathbb{R} \cup\{+\infty\}$, we denote by dom $(f)=\left\{x \in \mathbb{R}^{n}: f(x)<+\infty\right\}$, the effective domain of $f$. If $\operatorname{dom}(f) \neq \emptyset, f$ is called proper. If $\lim _{\|x\| \rightarrow+\infty} f(x)=+\infty, f$ is called coercive. We denote by $\arg \min \left\{f(x): x \in \mathbb{R}^{n}\right\}$ the set of minimizer of the function $f$ and by $f *$, the optimal value of problem: $\min \left\{f(x): x \in \mathbb{R}^{n}\right\}$, if it exists. The function $f$ is lower semicontinuous at $\bar{x}$ if for all sequence $\left\{x_{k}\right\}_{k \in \mathbb{N}}$ such that $\lim _{k \rightarrow+\infty} x_{k}=\bar{x}$ we obtain that $f(\bar{x}) \leq \liminf _{k \rightarrow+\infty} f\left(x_{k}\right)$.

Definition 2.1.1 Let $f: \mathbb{R}^{n} \longrightarrow \mathbb{R} \cup\{+\infty\}$ be a proper function. We say that $f$ is locally Lipschitz at $x \in \operatorname{dom}(f)$ if there exists $\varepsilon_{x}>0$ such that

$$
|f(z)-f(y)| \leq L_{x}\|z-y\|, \forall z, y \in B\left(x, \varepsilon_{x}\right) \cap \operatorname{dom}(f),
$$

where $B\left(x, \varepsilon_{x}\right)=\left\{y \in \mathbb{R}^{n}:\|y-x\|<\varepsilon_{x}\right\}$ and $L_{x}$ is some positive number. $f$ is locally Lipschitz on $\mathbb{R}^{n}$ if $f$ is locally Lipschitz for each $x \in \operatorname{dom}(f)$

The next result ensures that the set of minimizers of a function, under some assumptions, is nonempty. 


\section{Proposition 2.1.1 (Rockafellar and Wets [29], Theorem 1.9)}

Suppose that $f: \mathbb{R}^{n} \longrightarrow \mathbb{R} \cup\{+\infty\}$ is proper, lower semicontinuous and coercive, then the optimal value $f^{*}$ is finite and the set $\arg \min \left\{f(x): x \in \mathbb{R}^{n}\right\}$ is nonempty and compact.

Definition 2.1.2 Let $D \subset \mathbb{R}^{n}$ and $\bar{x} \in D$. The normal cone at the point $\bar{x}$ related to the set $D$ is given by $\mathcal{N}_{D}(\bar{x})=\left\{v \in \mathbb{R}^{n}:\langle v, x-\bar{x}\rangle \leq 0, \forall x \in D\right\}$.

\subsection{Multiobjective optimization}

In this subsection we present some properties and notation on multiobjective optimization. Those basic facts can be seen, for example, in Miettinen [22] and Luc [18].

Throughout this paper we consider the cone $\mathbb{R}_{+}^{m}=\left\{y \in \mathbb{R}^{m}: y_{i} \geq 0, \forall i=1, \ldots, m\right\}$, which induce a partial order $\preceq$ in $\mathbb{R}^{m}$ given by, for $y, y^{\prime} \in \mathbb{R}^{m}, y \preceq y^{\prime}$ if, and only if, $y^{\prime}-y$ $\in \mathbb{R}_{+}^{m}$, this means that $y_{i} \leq y_{i}^{\prime}$ for all $i=1,2, \ldots, m$. Given $\mathbb{R}_{++}^{m}$ the above relation induce the following one $\prec$, induced by the interior of this cone, given by, $y \prec y^{\prime}$, if, and only if, $y^{\prime}-y$ $\in \mathbb{R}_{++}^{m}$, this means that $y_{i}<y_{i}^{\prime}$ for all $i=1,2, \ldots, m$. Those partial orders establish a class of problems known in the literature as Multiobjective Optimization.

Let us consider the unconstrained multiobjective optimization problem (MOP) :

$$
\min \left\{G(x): x \in \mathbb{R}^{n}\right\}
$$

where $G: \mathbb{R}^{n} \longrightarrow \mathbb{R}^{m}$, with $G=\left(G_{1}, G_{2}, \ldots, G_{m}\right)^{T}$.

Definition 2.2.1 (Miettinen [22], Definition 2.2.1) A point $x^{*} \in \mathbb{R}^{n}$ is a Pareto solution of the problem (21), if there does not exist $x \in \mathbb{R}^{n}$ such that $G_{i}(x) \leq G_{i}\left(x^{*}\right)$, for all $i \in\{1, \ldots, m\}$ and $G_{j}(x)<G_{j}\left(x^{*}\right)$, for at least one index $j \in\{1, \ldots, m\}$.

Definition 2.2.2 (Miettinen [22],Definition 2.5.1) A point $x^{*} \in \mathbb{R}^{n}$ is a weak Pareto solution of the problem (2), if there does not exist $x \in \mathbb{R}^{n}$ such that $G_{i}(x)<G_{i}\left(x^{*}\right)$, for all $i \in\{1, \ldots, m\}$.

We denote by $\arg \min \left\{G(x): x \in \mathbb{R}^{n}\right\}$ and by arg $\min _{w}\left\{G(x): x \in \mathbb{R}^{n}\right\}$ the set of Pareto solutions and weak Pareto solutions to the problem (2), respectively. It is easy to check that $\arg \min \left\{G(x): x \in \mathbb{R}^{n}\right\} \subset \arg \min _{w}\left\{G(x): x \in \mathbb{R}^{n}\right\}$.

\subsection{Quasiconvex and Convex Functions}

In this subsection we present the concept and characterization of quasiconvex functions and quasiconvex multiobjective function. This theory can be found in Bazaraa et al. 2], Luc [18], Mangasarian [19], and their references.

Definition 2.3.1 Let $f: \mathbb{R}^{n} \longrightarrow \mathbb{R} \cup\{+\infty\}$ be a proper function. Then, $f$ is called quasiconvex if for all $x, y \in \mathbb{R}^{n}$, and for all $t \in[0,1]$, it holds that $f(t x+(1-t) y) \leq \max \{f(x), f(y)\}$.

Definition 2.3.2 Let $f: \mathbb{R}^{n} \longrightarrow \mathbb{R} \cup\{+\infty\}$ be a proper function. Then, $f$ is called convex if for all $x, y \in \mathbb{R}^{n}$, and for all $t \in[0,1]$, it holds that $f(t x+(1-t) y) \leq t f(x)+(1-t) f(y)$. 
Observe that if $f$ is a quasiconvex function then $\operatorname{dom}(f)$ is a convex set. On the other hand, while a convex function can be characterized by the convexity of its epigraph, a quasiconvex function can be characterized by the convexity of the lower level sets:

Definition 2.3.3 (Luc [18], Corollary 6.6) Let $F=\left(F_{1}, \ldots, F_{m}\right)^{T}: \mathbb{R}^{n} \longrightarrow \mathbb{R}^{m}$ be a function, then $F$ is $\mathbb{R}_{+}^{m}$ - quasiconvex if and only if every component function of $F, F_{i}: \mathbb{R}^{n} \longrightarrow$ $\mathbb{R}$, is quasiconvex.

Definition 2.3.4 Let $F=\left(F_{1}, \ldots, F_{m}\right)^{T}: \mathbb{R}^{n} \longrightarrow \mathbb{R}^{m}$ be a function, then $F$ is $\mathbb{R}_{+}^{m}$ - convex if and only if every component function of $F, F_{i}: \mathbb{R}^{n} \longrightarrow \mathbb{R}$, is convex.

Definition 2.3.5 Let $F=\left(F_{1}, \ldots, F_{m}\right)^{T}: \mathbb{R}^{n} \longrightarrow \mathbb{R}^{m}$ be a function, then $F$ is locally Lipschitz on $\mathbb{R}^{n}$ if and only if every component function of $F, F_{i}: \mathbb{R}^{n} \longrightarrow \mathbb{R}$, is locally Lipschitz on $\mathbb{R}^{n}$.

\subsection{Fréchet and Limiting Subdifferentials}

Definition 2.4.1 Let $f: \mathbb{R}^{n} \rightarrow \mathbb{R} \cup\{+\infty\}$ be a proper function.

(a) For each $x \in \operatorname{dom}(f)$, the set of regular subgradients (also called Fréchet subdifferential) of $f$ at $x$, denoted by $\hat{\partial} f(x)$, is the set of vectors $v \in \mathbb{R}^{n}$ such that

$$
f(y) \geq f(x)+\langle v, y-x\rangle+o(\|y-x\|), \text { where } \lim _{y \rightarrow x} \frac{o(\|y-x\|)}{\|y-x\|}=0 .
$$

Or equivalently, $\hat{\partial} f(x):=\left\{v \in \mathbb{R}^{n}: \liminf _{y \neq x, y \rightarrow x} \frac{f(y)-f(x)-\langle v, y-x\rangle}{\|y-x\|} \geq 0\right\}$. If $x \notin \operatorname{dom}(f)$ then $\hat{\partial} f(x)=\emptyset$.

(b) The set of general subgradients (also called limiting subdifferential) $f$ at $x \in \mathbb{R}^{n}$, denoted by $\partial f(x)$, is defined as follows:

$$
\partial f(x):=\left\{v \in \mathbb{R}^{n}: \exists x_{n} \rightarrow x, \quad f\left(x_{n}\right) \rightarrow f(x), \quad v_{n} \in \hat{\partial} f\left(x_{n}\right) \text { and } v_{n} \rightarrow v\right\} .
$$

Proposition 2.4.1 For a function $f: \mathbb{R}^{n} \rightarrow \mathbb{R} \cup\{+\infty\}$ and a point $\bar{x} \in \operatorname{dom}(f)$, the subgradient sets $\partial f(\bar{x})$ and $\hat{\partial} f(\bar{x})$ are closed, with $\hat{\partial} f(\bar{x})$ convex and $\hat{\partial} f(\bar{x}) \subset \partial f(\bar{x})$.

Proof. See Rockafellar and Wets [29], Theorem 8.6.

Proposition 2.4.2 (Fermat's rule generalized) If a proper function $f: \mathbb{R}^{n} \rightarrow \mathbb{R} \cup\{+\infty\}$ has a local minimum at $\bar{x} \in \operatorname{dom}(f)$, then $0 \in \hat{\partial} f(\bar{x})$.

Proof. See Rockafellar and Wets [29], Theorem 10.1.

Proposition 2.4.3 Let $f, g: \mathbb{R}^{n} \rightarrow \mathbb{R} \cup\{+\infty\}$ proper functions such that $f$ is locally Lipschitz at $\bar{x} \in \operatorname{dom}(f) \cap \operatorname{dom}(g)$ and $g$ is lower semicontinuous function at this point. Then,

$$
\partial(f+g)(\bar{x}) \subset \partial f(\bar{x})+\partial g(\bar{x})
$$

Proof. See Mordukhovich 23] Theorem 2.33. 


\subsection{Clarke Subdifferential}

Definition 2.5.1 Let $f: \mathbb{R}^{n} \rightarrow \mathbb{R} \cup\{+\infty\}$ be a proper locally Lipschitz function at $x \in \operatorname{dom}(f)$ and $d \in \mathbb{R}^{n}$. The Clarke directional derivative of $f$ at $x$ in the direction $d$, denoted by $f^{o}(x, d)$, is defined as

$$
f^{o}(x, d)=\limsup _{t \downarrow 0} \frac{f(y+t d)-f(y)}{t}
$$

and the Clarke subdifferential of $f$ at $x$, denoted by $\partial^{o} f(x)$, is defined as

$$
\partial^{o} f(x)=\left\{w \in \mathbb{R}^{n}:\langle w, d\rangle \leq f^{o}(x, d), \forall d \in \mathbb{R}^{n}\right\} .
$$

Remark 2.5.1 From the above definitions it follows directly that for all $x \in \mathbb{R}^{n}$, one has $\hat{\partial} f(x) \subset \partial f(x) \subset \partial^{\circ} f(x)$ (see Bolte et al. [4], Inclusion (7)).

Lemma 2.5.1 Let $f, g: \mathbb{R}^{n} \rightarrow \mathbb{R} \cup\{+\infty\}$ be locally Lipschitz functions at $x \in \mathbb{R}^{n}$. Then, $\forall d \in \mathbb{R}^{n}$ :

(i) $(f+g)^{o}(x, d) \leq f^{o}(x, d)+g^{o}(x, d)$;

(ii) $(\lambda f)^{o}(x, d)=\lambda\left(f^{o}(x, d)\right), \forall \lambda \geq 0$;

(iii) $f^{o}(x, \lambda d)=\lambda f^{o}(x, d), \forall \lambda \geq 0$.

Proof. It is immediate from Clarke directional derivative.

Lemma 2.5.2 Let $f: \mathbb{R}^{n} \rightarrow \mathbb{R}$ be locally Lipschitz function at $x$ and any scalar $\lambda$, then

$$
\partial^{o}(\lambda f)(x) \subset \lambda \partial^{o} f(x)
$$

Proof. See Clarke [8], Proposition 2.3.1.

Lemma 2.5.3 Let $f_{i}: \mathbb{R}^{n} \rightarrow \mathbb{R} \cup\{+\infty\}, i=1,2, \ldots, m$, be locally Lipschitz functions at $x$, then

$$
\partial^{o}\left(\sum_{i=1}^{m} f_{i}\right)(x) \subset \sum_{i=1}^{m} \partial^{o} f_{i}(x)
$$

Proof. See Clarke [8], Proposition 2.3.3.

Proposition 2.5.1 Let $f: \mathbb{R}^{n} \rightarrow \mathbb{R} \cup\{+\infty\}$ be a proper locally Lipschitz function on $\mathbb{R}^{n}$. Then, $f^{o}$ is upper semicontinuous, i.e, if $\left\{\left(x^{k}, d^{k}\right)\right\}$ is a sequence in $\mathbb{R}^{n} \times \mathbb{R}^{n}$ such that $\lim _{k \rightarrow+\infty}\left(x^{k}, d^{k}\right)=$ $(x, d)$ then $\limsup _{k \rightarrow+\infty} f^{o}\left(x^{k}, d^{k}\right) \leq f^{o}(x, d)$.

Proof. See Clarke [8], Proposition 2.1.1, (b). 
Proposition 2.5.2 Let $f: \mathbb{R}^{n} \longrightarrow \mathbb{R}$ be a quasiconvex locally Lipschitz function on $\mathbb{R}^{n}$. If $g \in \partial^{\circ} f(x)$, such that $\langle g, \tilde{x}-x\rangle>0$ then, $f(x) \leq f(\tilde{x})$.

Proof. See Aussel [1], Theorem 2.1.

Proposition 2.5.3 Let $f: \mathbb{R}^{n} \longrightarrow \mathbb{R}$ be a convex function. Then $\partial^{o} f(x)$ coincides with the subdifferential at $x$ in the sense of convex analysis, and $f^{o}(x, d)$ coincides with the directional derivative $f^{\prime}(x, d)$ for each $d$.

Proof. See Clarke [8], Proposition 2.2.7

\subsection{Descent direction}

We are now able to introduce the definition of Pareto-Clarke critical point for locally Lipschitz functions on $\mathbb{R}^{n}$, which will play a key role in our paper.

Definition 2.6.1 (Custódio et al. [9], Definition 4.6) Let $F=\left(F_{1}, \ldots, F_{m}\right)^{T}: \mathbb{R}^{n} \longrightarrow \mathbb{R}^{m}$ be locally Lipschitz on $\mathbb{R}^{n}$. We say that $x^{*} \in \mathbb{R}^{n}$ is a Pareto-Clarke critical point of $F$ if, for all directions $d \in \mathbb{R}^{n}$, there exists $i_{0}=i_{0}(d) \in\{1, \ldots, m\}$ such that $F_{i_{o}}^{o}\left(x^{*}, d\right) \geq 0$.

Definition 2.6.1 says essentially that there is no direction in $\mathbb{R}^{n}$ that is descent for all the objective functions (see, for instance, (Custódio et al. 9]). If a point is a Pareto minimizer (local or global), then it is necessarily a Pareto-Clarke critical point .

Remark 2.6.1 Follows from the previous definition that, if a point $x$ is not Pareto-Clarke critical, there exists a direction $d \in \mathbb{R}^{n}$ satisfying

$$
F_{i}^{o}(x, d)<0, \forall i \in\{1, \ldots, m\}
$$

This implies that, for each $i \in\{1, \ldots, m\}, d$ is a descent direction, for each function $F_{i}$, i.e, there exists $\varepsilon>0$, such that

$$
F_{i}(x+t d)<F_{i}(x), \forall t \in(0, \varepsilon], \forall i \in\{1, \ldots, m\} .
$$

It is a well known fact that such $d$ is a descent direction for the multiobjective function $F$ at $x$, i.e, $\exists \varepsilon>0$ such that

$$
F(x+t d) \prec F(x), \forall t \in(0, \varepsilon] .
$$

Proposition 2.6.1 Let $\bar{x}$ be a Pareto-Clarke critical point of a locally Lipschitz $G: \mathbb{R}^{n} \longrightarrow$

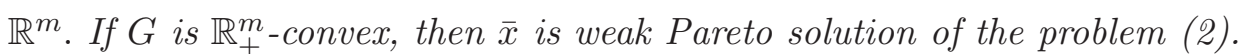

Proof. As $\bar{x}$ is a Pareto-Clarke critical point of $G$ then for all directions $d$ there exists $i_{0}=i_{0}(d) \in\{1, \ldots, m\}$ such that $G_{i_{o}}^{o}(\bar{x}, d) \geq 0$. Now, due that $G$ is $\mathbb{R}_{+}^{m}-$ convex then the last is equivalent, see Proposition 2.5.3, to

$$
G_{i_{o}}^{\prime}(\bar{x}, d) \geq 0
$$


where $G_{i_{0}}^{\prime}(\bar{x}, d)$ is the directional derivative of the convex function $G_{i_{0}}$ at $\bar{x}$ in the direction $d$. On the other hand, suppose by contradiction that $\bar{x}$ is not a weak Pareto solution of the problem (22), then exists $x^{*} \in \mathbb{R}^{n}$ such that

$$
G\left(x^{*}\right) \prec G(\bar{x}) \text {, i.e, } G_{i}\left(x^{*}\right)<G_{i}(\bar{x}), \forall i \in 1, \ldots, m \text {. }
$$

Thus, for all $i$, there exists $\alpha=\alpha(i)>0$ such that $G_{i}\left(x^{*}\right)=G_{i}(\bar{x})-\alpha$. Define $x_{\lambda}=$ $\lambda x^{*}+(1-\lambda) \bar{x}, \lambda \in(0,1)$. From the $\mathbb{R}_{+}^{m}$-convexity of $G$ we have

$$
G_{i}\left(x_{\lambda}\right)=G_{i}\left(\lambda x^{*}+(1-\lambda) \bar{x}\right) \leq \lambda G_{i}\left(x^{*}\right)+(1-\lambda) G_{i}(\bar{x})=-\alpha \lambda+G_{i}(\bar{x})
$$

Its follows that

$$
\frac{G_{i}\left(\bar{x}+\lambda\left(x^{*}-\bar{x}\right)\right)-G_{i}(\bar{x})}{\lambda} \leq-\alpha<0, \forall \lambda \in(0,1) .
$$

Taking $\bar{d}=x^{*}-\bar{x} \in \mathbb{R}^{n}$ and limit when $\lambda$ converges to zero in the above inequality we obtain a contradiction with (3)). Therefore $\bar{x}$ is a weak Pareto solution of the problem (2).

\subsection{Fejér convergence}

Definition 2.7.1 A seguence $\left\{y_{k}\right\} \subset \mathbb{R}^{n}$ is said to be Fejér convergent to a set $U \subseteq \mathbb{R}^{n}$ if, $\left\|y_{k+1}-u\right\| \leq\left\|y_{k}-u\right\|, \forall k \in \mathbb{N}, \forall u \in U$.

The following result on Fejér convergence is well known.

Lemma 2.7.1 If $\left\{y_{k}\right\} \subset \mathbb{R}^{n}$ is Fejér convergent to some set $U \neq \emptyset$, then:

(i) The sequence $\left\{y_{k}\right\}$ is bounded.

(ii) If an accumulation point $y$ of $\left\{y_{k}\right\}$ belongs to $U$, then $\lim _{k \rightarrow+\infty} y_{k}=y$.

Proof. See Schott [30, Theorem 2.7.

\section{Scalarization proximal point method (SPPM)}

We are interested in solving the unconstrained multiobjective optimization problem (MOP):

$$
\min \left\{F(x): x \in \mathbb{R}^{n}\right\}
$$

where $F: \mathbb{R}^{n} \rightarrow \mathbb{R}^{m}$ is a vector function satisfying the following assumptions:

$\left(\mathbf{H}_{1}\right) \quad F$ is locally Lipschitz on $\mathbb{R}^{n}$.

$\left(\mathbf{H}_{\mathbf{2}}\right) \quad F$ is $\mathbb{R}_{+}^{m}$-quasiconvex. 


\subsection{The algorithm}

In this subsection, we propose a Scalarization Proximal Point Method with quadratic regularization, denoted by SPPM, to solve the problem (4).

\section{SPPM Algorithm}

Initialization: Choose an arbitrary initial point

$$
x^{0} \in \mathbb{R}^{n}
$$

Main Steps: Given $x^{k}$, find $x^{k+1} \in \Omega_{k}$ such that

$$
0 \in \partial^{o}\left(\left\langle F(.), z_{k}\right\rangle+\frac{\alpha_{k}}{2}\left\langle e_{k}, z_{k}\right\rangle\left\|\cdot-x^{k}\right\|^{2}\right)\left(x^{k+1}\right)+\mathcal{N}_{\Omega_{k}}\left(x^{k+1}\right)
$$

where $\Omega_{k}=\left\{x \in \mathbb{R}^{n}: F(x) \preceq F\left(x^{k}\right)\right\}, \alpha_{k}>0,\left\{e_{k}\right\} \subset \mathbb{R}_{++}^{m},\left\|e_{k}\right\|=1,\left\{z_{k}\right\} \subset \mathbb{R}_{+}^{m} \backslash\{0\}$ and $\left\|z_{k}\right\|=1$.

Stop Criterion: If $x^{k+1}=x^{k}$ or $x^{k+1}$ is a Pareto-Clarke critical point, then stop. Otherwise, to do $k \leftarrow k+1$ and return to Main Steps.

Remark 3.1.1 If $F$ is $\mathbb{R}_{+}^{n}$-convex the main step (6) is equivalent to:

$$
x^{k+1}=\operatorname{argmin}\left\{\left\langle F(x), z_{k}\right\rangle+\frac{\alpha_{k}}{2}\left\langle e_{k}, z_{k}\right\rangle\left\|x-x^{k}\right\|^{2}: x \in \Omega_{k}\right\}
$$

This iteration has been studied by Bonnel et al. [5], so we can say that, in certain sense, our iteration is an extension for the nonconvex case of that work. On the other hand, when $F$ is $\mathbb{R}_{+}^{n}$-quasiconvex, the regularized function $F_{k}=\left\langle F(x), z_{k}\right\rangle+\frac{\alpha_{k}}{2}\left\langle e_{k}, z_{k}\right\rangle\left\|x-x^{k}\right\|^{2}$ is not necessarily quasiconvex and so (7) is a global optimization problem, it is the reason for which we consider the more weak iteration (6).

\subsection{Existence of the iterates}

Theorem 3.2.1 Let $F: \mathbb{R}^{n} \longrightarrow \mathbb{R}^{m}$ be a function satisfying $\left(\mathbf{H}_{\mathbf{1}}\right),\left(\mathbf{H}_{\mathbf{2}}\right)$ and $0 \prec F$. Then the sequence $\left\{x^{k}\right\}$, generated by the SPPM algorithm, given by (5) and (6), is well defined.

Proof. We proceed by induction. It holds for $k=0$, due to (51). Assume that $x^{k}$ exists and define $\varphi_{k}(x)=\left\langle F(x), z_{k}\right\rangle+\frac{\alpha_{k}}{2}\left\langle e_{k}, z_{k}\right\rangle\left\|x-x^{k}\right\|^{2}+\delta_{\Omega_{k}}(x)$, where $\delta_{\Omega_{k}}($.$) is the indicator$ function of $\Omega_{k}$. Then we have that $\min \left\{\varphi_{k}(x): x \in \mathbb{R}^{n}\right\}$ is equivalent to $\min \left\{\left\langle F(x), z_{k}\right\rangle+\right.$ $\left.\frac{\alpha_{k}}{2}\left\langle e_{k}, z_{k}\right\rangle\left\|x-x^{k}\right\|^{2}: x \in \Omega_{k}\right\}$. Due that $0 \prec F$ and $z_{k} \in \mathbb{R}_{+}^{m} \backslash\{0\}$ the function $\left\langle F(),. z_{k}\right\rangle$ is bounded from below. Then, by the lower boundedness and continuity of the function $\left\langle F(),. z_{k}\right\rangle$, as also, by the continuity and coercivity of $\left\|.-x^{k}\right\|^{2}$, and using Proposition 2.1.1, we obtain that there exists $x^{k+1} \in \Omega_{k}$ which is a global minimum of $\varphi_{k}($.$) . From Proposition 2.4.2, x^{k+1}$ satisfies $0 \in \hat{\partial}\left(\left\langle F(),. z_{k}\right\rangle+\frac{\alpha_{k}}{2}\left\langle e_{k}, z_{k}\right\rangle\left\|\cdot-x^{k}\right\|^{2}+\delta_{\Omega_{k}}().\right)\left(x^{k+1}\right)$ and by Proposition 2.4.1 and Proposition 2.4 .3 , we have that

$$
0 \in \partial\left(\left\langle F(.), z_{k}\right\rangle+\frac{\alpha_{k}}{2}\left\langle e_{k}, z_{k}\right\rangle\left\|\cdot-x^{k}\right\|^{2}\right)\left(x^{k+1}\right)+\mathcal{N}_{\Omega_{k}}\left(x^{k+1}\right)
$$


From Remark 2.5.1, the iteration (6) is obtained of (8).

Remark 3.2.1 (Huang and Yang [15]) Without loss of generality, always we can assume that the function $F: \mathbb{R}^{n} \longrightarrow \mathbb{R}^{m}$ satisfies $0 \prec F$. Of fact, consider the following multiobjective optimization problem

$$
\left(P^{\prime}\right) \min \left\{e^{F(x)}: x \in \mathbb{R}^{n}\right\}
$$

Observe that both, (4) and $\left(P^{\prime}\right)$, have the same set of Pareto solutions, weak Pareto solutions and Pareto-Clarke critical points. Furthermore, if $F$ is $\mathbb{R}_{+}^{m}-$ quasiconvex and locally Lipschitz on $\mathbb{R}^{n}$, then $e^{F(x)}$ is also $\mathbb{R}_{+}^{m}$ - quasiconvex and locally Lipschitz on $\mathbb{R}^{n}$. Therefore, along this paper and from now on we implicitly assume that $0 \prec F$.

Remark 3.2.2 We are interest in the asymptotic convergence of the (SPPM) algorithm, so we also assume along this paper that in each iteration $x^{k}$ is not a Pareto-Clarke critical point and $x^{k+1} \neq x^{k}$. This implies, from Remark 2.6.1 that the interior of $\Omega_{k+1}$, denoted by $\Omega_{k+1}^{0}$, is nonempty.

When the condition $x^{k+1}=x^{k}$ is not satisfied, that is, if there exists $k_{0}$ such that $x^{k_{0}+1}=$ $x^{k_{0}}$ then it is easy to prove that this point is a Pareto-Clarke critical point of F.

\subsection{Weak Convergence}

In this subsection we prove, under the assumption that the consecutive iterations converges to zero, that any cluster point is a Pareto-Clarke critical point of the problem (44).

Proposition 3.3.1 Let $F: \mathbb{R}^{n} \longrightarrow \mathbb{R}^{m}$ be a function satisfying $\left(\mathbf{H}_{\mathbf{1}}\right)$ and $\left(\mathbf{H}_{\mathbf{2}}\right)$. If $0<\alpha_{k}<\tilde{\alpha}$, with $\tilde{\alpha}>0$, and the sequence $\left\{x^{k}\right\}$ generated by the SPPM algorithm, (5) and (6), satisfies

$$
\lim _{k \rightarrow+\infty}\left\|x^{k+1}-x^{k}\right\|=0
$$

and has a cluster point, then it is a Pareto-Clarke critical point of the problem (4).

Proof. By assumption, there exists a convergent subsequence $\left\{x^{k_{j}}\right\}$ of $\left\{x^{k}\right\}$ whose limit is some $\widehat{x} \in \mathbb{R}^{n}$. Since $F$ is locally Lipschitz on $\mathbb{R}^{n}$, then the function $\langle F(), z$.$\rangle is also lo-$ cally Lipschitz on $\mathbb{R}^{n}$ and so, continuos for all $z \in \mathbb{R}^{m}$, in particular, for all $z \in \mathbb{R}_{+}^{m} \backslash\{0\}$, and $\lim _{j \rightarrow+\infty}\left\langle F\left(x^{k_{j}}\right), z\right\rangle=\langle F(\widehat{x}), z\rangle$. On the other hand, as $x^{k+1} \in \Omega_{k}$, we have $F\left(x^{k+1}\right) \preceq$ $F\left(x^{k}\right)$ and since $z \in \mathbb{R}_{+}^{m} \backslash\{0\}$, we conclude that the sequence $\left\{\left\langle F\left(x^{k}\right), z\right\rangle\right\}$ is convergent to $\langle F(\widehat{x}), z\rangle$ because it is nonincreasing and admits a subsequence converging to $\langle F(\widehat{x}), z\rangle$. So $\lim _{k \rightarrow+\infty}\left\langle F\left(x^{k}\right), z\right\rangle=\langle F(\widehat{x}), z\rangle=i n f_{k \in \mathbb{N}}\left\{\left\langle F\left(x^{k}\right), z\right\rangle\right\} \leq\left\langle F\left(x^{k}\right), z\right\rangle$. Thus, $\left\langle F\left(x^{k}\right)-F(\widehat{x}), z\right\rangle \geq$ $0, \forall k \in \mathbb{N}, \forall z \in \mathbb{R}_{+}^{m} \backslash\{0\}$. We conclude that $F\left(x^{k}\right)-F(\widehat{x}) \in \mathbb{R}_{+}^{m}$, i.e, $F(\widehat{x}) \preceq F\left(x^{k}\right), \forall k \in \mathbb{N}$. This implies that $\widehat{x} \in \Omega_{k}$.

Assume, to arrive at a contradiction, that $\widehat{x}$ is not Pareto-Clarke critical point in $\mathbb{R}^{n}$, then there exists a direction $d \in \mathbb{R}^{n}$ such that

$$
F_{i}^{o}(\widehat{x}, d)<0, \forall i \in\{1, \ldots, m\}
$$

Therefore $d$ is a descent direction for the multiobjective function $F$ in $\widehat{x}$, so, $\exists \varepsilon>0$ such that $F(\widehat{x}+\lambda d) \prec F(\widehat{x}), \forall \lambda \in(0, \varepsilon]$. Thus, $\widehat{x}+\lambda d \in \Omega_{k}$. 
On the other hand, as $\left\{x^{k}\right\}$ is generated by SPPM algorithm, from Theorem 3.2.1, (6), Lemma 2.5.3 and from Lemma 2.5.2, this implies that there exists $\beta_{k}\left(x^{k}-x^{k+1}\right)-v_{k} \in$ $\partial^{o}\left(\left\langle F(),. z_{k}\right\rangle\right)\left(x^{k+1}\right)$, with $v_{k} \in \mathcal{N}_{\Omega_{k}}\left(x^{k+1}\right)$ and $\beta_{k}=\alpha_{k}\left\langle e_{k}, z_{k}\right\rangle>0$, such that

$$
\beta_{k}\left\langle x^{k}-x^{k+1}, p\right\rangle-\left\langle v_{k}, p\right\rangle \leq\left\langle F(.), z_{k}\right\rangle^{o}\left(x^{k+1}, p\right), \forall p \in \mathbb{R}^{n}
$$

Consider $p=(\widehat{x}+\lambda d)-x^{k+1}$ and as $v_{k} \in \mathcal{N}_{\Omega_{k}}\left(x^{k+1}\right)$, from (11) we have

$$
\beta_{k}\left\langle x^{k}-x^{k+1}, \widehat{x}+\lambda d-x^{k+1}\right\rangle \leq\left\langle F(.), z_{k}\right\rangle^{o}\left(x^{k+1}, \widehat{x}+\lambda d-x^{k+1}\right)
$$

As $\left\{z_{k}\right\}$ is bounded, then there exists a subsequence denoted also, without loss of generality, by $\left\{z^{k_{j}}\right\}$ such that $\lim _{j \rightarrow+\infty} z^{k_{j}}=\bar{z}$, with $\bar{z} \in \mathbb{R}_{+}^{m} \backslash\{0\}$. From (12), we have:

$$
\beta_{k_{j}}\left\langle x^{k_{j}}-x^{k_{j}+1}, \widehat{x}+\lambda d-x^{k_{j}+1}\right\rangle \leq\left\langle F(.), z_{k_{j}}\right\rangle^{o}\left(x^{k_{j}+1}, \widehat{x}+\lambda d-x^{k_{j}+1}\right)
$$

Lemma 2.5.1, (i) and (ii), we have:

$$
\beta_{k_{j}}\left\langle x^{k_{j}}-x^{k_{j}+1}, \widehat{x}+\lambda d-x^{k_{j}+1}\right\rangle \leq \sum_{i=1}^{m} z_{k_{j}}^{i} F_{i}^{0}\left(x^{k_{j}+1}, \widehat{x}+\lambda d-x^{k_{j}+1}\right),
$$

where $z_{k_{j}}^{i}$ are the components of the vector $z_{k_{j}}$. Then using Lemma 2.5.1, (iii), we obtain:

$$
\beta_{k_{j}}\left\langle x^{k_{j}}-x^{k_{j}+1}, \widehat{x}+\lambda d-x^{k_{j}+1}\right\rangle \leq \sum_{i=1}^{m} F_{i}^{0}\left(x^{k_{j}+1}, z_{k_{j}}^{i}\left(\widehat{x}+\lambda d-x^{k_{j}+1}\right)\right),
$$

Taking lim sup in the above inequality, using the condition (9), Proposition 2.5.1 and as $\lambda>0$, we conclude that

$$
0 \leq F_{1}^{o}(\widehat{x}, d) \bar{z}_{1}+\ldots+F_{m}^{o}(\widehat{x}, d) \bar{z}_{m}
$$

Without loss of generality, consider the set $J=\left\{i \in I: \bar{z}_{i}>0\right\}$, where $I=\{1, \ldots, m\}$. Thus, from (13), there exists $i_{0} \in J$ such that $F_{i_{0}}^{o}(\widehat{x}, d) \bar{z}_{i_{0}} \geq 0$ contradicting (10).

\subsection{Global Convergence}

For this subsection we make the following assumption on the function $F$ and the initial point $x^{0}:$

$\left(\mathbf{H}_{3}\right)$ The set $\left(F\left(x^{0}\right)-\mathbb{R}_{+}^{m}\right) \cap F\left(\mathbb{R}^{n}\right)$ is $\mathbb{R}_{+}^{m}$ - complete, meaning that for all sequences $\left\{a_{k}\right\} \subset$ $\mathbb{R}^{n}$, with $a_{0}=x^{0}$, such that $F\left(a_{k+1}\right) \preceq F\left(a_{k}\right)$, there exists $a \in \mathbb{R}^{n}$ such that $F(a) \preceq$ $F\left(a_{k}\right), \forall k \in \mathbb{N}$.

Remark 3.4.1 The assumption $\left(\mathbf{H}_{\mathbf{3}}\right)$ is cited in various works on proximal point method for convex functions, see Bonnel et al. [5], Ceng and Yao [7] and Villacorta and Oliveira [31].

As the sequence $\left\{x^{k}\right\}$ generated by SPPM algorithm, satisfies the assumption $\left(\mathbf{H}_{\mathbf{3}}\right)$ and from assumptions $\left(\mathbf{H}_{\mathbf{1}}\right)$ and $\left(\mathbf{H}_{\mathbf{2}}\right)$ then

$$
E=\left\{x \in \mathbb{R}^{n}: F(x) \preceq F\left(x^{k}\right), \quad \forall k \in \mathbb{N}\right\}
$$


is a nonempty closed convex set.

Proposition 3.4.1 (Fejér convergence)

Under assumptions $\left(\mathbf{H}_{\mathbf{1}}\right),\left(\mathbf{H}_{\mathbf{2}}\right)$ and $\left(\mathbf{H}_{\mathbf{3}}\right)$, the sequence $\left\{x^{k}\right\}$ generated by the SPPM algorithm, (5) and (6), is Fejér convergent to $E$.

Proof. From Theorem 3.2.1, (6), Lemma 2.5.3 and from Lemma 2.5.2 we obtain that there exist $g_{i}^{k} \in \partial^{o} F_{i}\left(x^{k+1}\right), i=1, \ldots, m$ such that

$$
0 \in \sum_{i=1}^{m} z_{k}^{i} g_{i}^{k}+\alpha_{k}\left\langle e_{k}, z_{k}\right\rangle\left(x^{k+1}-x^{k}\right)+\mathcal{N}_{\Omega_{k}}\left(x^{k+1}\right)
$$

where $z_{k}^{i}$ are the components of $z_{k}$. Thus there exist vectors $g_{i}^{k} \in \partial^{o} F_{i}\left(x^{k+1}\right), i=1, \ldots, m$, and $v_{k} \in \mathcal{N}_{\Omega_{k}}\left(x^{k+1}\right)$ such that

$$
\sum_{i=1}^{m} z_{k}^{i} g_{i}^{k}=\beta_{k}\left(x^{k}-x^{k+1}\right)-v_{k}
$$

where $\beta_{k}=\alpha_{k}\left\langle e_{k}, z_{k}\right\rangle, \forall k \in \mathbb{N}$. Note that $\beta_{k}>0$, because $\alpha_{k}>0, e_{k}$ belongs to $\mathbb{R}_{++}^{m}$, and $z_{k}$ belongs to $\mathbb{R}_{+}^{m} \backslash\{0\}$. From (14) we have

$$
x^{k}-x^{k+1}=\frac{1}{\beta_{k}}\left(\sum_{i=1}^{m} z_{k}^{i} g_{i}^{k}+v_{k}\right)
$$

Now take $x^{*} \in E$, then by definition of $E, x^{*} \in \Omega_{k+1}$ for all $k$, and from Remark 3.2.2, there exists $\left\{x^{l}\right\} \in \Omega_{k+1}^{0}$ such that $x^{l} \rightarrow x^{*}$. Observe that, $\forall x \in \mathbb{R}^{n}$ :

$$
\left\|x^{k}-x\right\|^{2}=\left\|x^{k}-x^{k+1}\right\|^{2}+\left\|x^{k+1}-x\right\|^{2}+2\left\langle x^{k}-x^{k+1}, x^{k+1}-x\right\rangle .
$$

Now,combining (16), with $x=x^{l}$, and (15), we have:

$$
\left\|x^{k}-x^{l}\right\|^{2}=\left\|x^{k}-x^{k+1}\right\|^{2}+\left\|x^{k+1}-x^{l}\right\|^{2}+\frac{2}{\beta_{k}}\left(\sum_{i=1}^{m} z_{k}^{i}\left\langle g_{i}^{k}, x^{k+1}-x^{l}\right\rangle+\left\langle v_{k}, x^{k+1}-x^{l}\right\rangle\right)
$$

As $F\left(x^{l}\right) \prec F\left(x^{k+1}\right)$, then $F_{i}\left(x^{l}\right)<F_{i}\left(x^{k+1}\right), \forall i=1, \ldots, m$. Furthermore, $g_{i}^{k} \in \partial^{o} F_{i}\left(x^{k+1}\right)$ and as $F_{i}$ is quasiconvex, using Proposition 2.5.2 we have

$$
\left\langle g_{i}^{k}, x^{k+1}-x^{l}\right\rangle \geq 0, \forall i=1, \ldots, m .
$$

Now, as $v_{k} \in \mathcal{N}_{\Omega_{k}}\left(x^{k+1}\right)$, the inequality (17) and (18), imply, taking $l \rightarrow \infty$

$$
0 \leq\left\|x^{k+1}-x^{k}\right\|^{2} \leq\left\|x^{k}-x^{*}\right\|^{2}-\left\|x^{k+1}-x^{*}\right\|^{2}, \forall k \in \mathbb{N}
$$

Thus,

$$
\left\|x^{k+1}-x^{*}\right\| \leq\left\|x^{k}-x^{*}\right\|
$$

Proposition 3.4.2 Under assumptions $\left(\mathbf{H}_{\mathbf{1}}\right),\left(\mathbf{H}_{\mathbf{2}}\right)$ and $\left(\mathbf{H}_{\mathbf{3}}\right)$, the sequence $\left\{x^{k}\right\}$ generates by the SPPM algorithm, (5) and (6), satisfies 


$$
\lim _{k \rightarrow+\infty}\left\|x^{k+1}-x^{k}\right\|=0 .
$$

Proof. It follows from (20), that $\forall x^{*} \in E,\left\{\left\|x^{k}-x^{*}\right\|\right\}$ is a nonnegative and nonincreasing sequence, and hence is convergent. Thus, the right-hand side of (19) converges to 0 as $k \rightarrow+\infty$, and the result is obtained.

Proposition 3.4.3 Under assumptions $\left(\mathbf{H}_{\mathbf{1}}\right),\left(\mathbf{H}_{\mathbf{2}}\right)$ and $\left(\mathbf{H}_{\mathbf{3}}\right)$, the sequence $\left\{x^{k}\right\}$ generated by the SPPM algorithm converges some point of $E$.

Proof. From Proposition 3.4.1 and Lemma 2.7.1, $(i),\left\{x^{k}\right\}$ is bounded, then exists a subsequence $\left\{x^{k_{j}}\right\}$ such that $\lim _{j \rightarrow+\infty} x^{k_{j}}=\widehat{x}$. Since $F$ is locally Lipschitz on $\mathbb{R}^{n}$, then the function $\langle F(), z$.$\rangle is also locally Lipschitz on \mathbb{R}^{n}$ and so, continuous for all $z \in \mathbb{R}^{m}$, in particular, for all $z \in \mathbb{R}_{+}^{m} \backslash\{0\}$, and $\lim _{j \rightarrow+\infty}\left\langle F\left(x^{k_{j}}\right), z\right\rangle=\langle F(\widehat{x}), z\rangle$. On the other hand, as $x^{k+1} \in \Omega_{k}$, we have $F\left(x^{k+1}\right) \preceq F\left(x^{k}\right)$ and since $z \in \mathbb{R}_{+}^{m} \backslash\{0\}$, we conclude $\left\langle F\left(x^{k+1}\right), z\right\rangle \leq\left\langle F\left(x^{k}\right), z\right\rangle$. Furthermore, from Remark 3.2.1, we can assume that the function $\langle F(), z$.$\rangle is bounded below,$ for each $z \in \mathbb{R}_{+}^{m} \backslash\{0\}$. Then the sequence $\left\{\left\langle F\left(x^{k}\right), z\right\rangle\right\}$ is nonincreasing and bounded below, hence convergent. So $\lim _{k \rightarrow+\infty}\left\langle F\left(x^{k}\right), z\right\rangle=\langle F(\widehat{x}), z\rangle=i n f_{k \in \mathbb{N}}\left\{\left\langle F\left(x^{k}\right), z\right\rangle\right\} \leq\left\langle F\left(x^{k}\right), z\right\rangle$. Thus, $\left\langle F\left(x^{k}\right)-F(\widehat{x}), z\right\rangle \geq 0, \forall k \in \mathbb{N}, \forall z \in \mathbb{R}_{+}^{m} \backslash\{0\}$. We conclude that $F\left(x^{k}\right)-F(\widehat{x}) \in \mathbb{R}_{+}^{m}$, i.e, $F(\widehat{x}) \preceq F\left(x^{k}\right), \forall k \in \mathbb{N}$. Thus $\widehat{x} \in E$, then using Lemma 2.7.1, (ii), we obtain the result.

Finally, we prove that the sequence of the iterations converges to a Pareto-Clarke critical point when the sequence of regularization parameters $\left\{\alpha_{k}\right\}$ is bounded.

Theorem 3.4.1 Consider $F: \mathbb{R}^{n} \longrightarrow \mathbb{R}^{m}$ a function satisfying the assumptions $\left(\mathbf{H}_{1}\right),\left(\mathbf{H}_{2}\right)$ and $\left(\mathbf{H}_{3}\right)$. If $0<\alpha_{k}<\tilde{\alpha}$, then the sequence $\left\{x_{k}\right\}$ generated by the SPPM algorithm, (5D) and (6)), converges to a Pareto-Clarke critical point of the problem (41).

Proof. From Proposition 3.4.3, $\left\{x^{k}\right\}$ converges, then this sequence has a unique cluster point $\bar{x}$ and from Proposition 3.4 .2 and Proposition 3.3 .1 we obtain the result.

Corollary 3.4.1 If $F: \mathbb{R}^{n} \longrightarrow \mathbb{R}^{m}$ is $\mathbb{R}_{+}^{m}$-convex and $\bar{x}$ the point of convergence given by the SPPM algorithm, given by (5) and (6), then $\bar{x}$ is weak Pareto solution of the problem (4).

Proof. It is inmediate from Proposition 2.6.1,

Corollary 3.4.2 If $F: \mathbb{R}^{n} \longrightarrow \mathbb{R}^{m}$ is continuously differentiable on $\mathbb{R}^{n}$ and satisfies the assumptions $\left(\mathbf{H}_{\mathbf{2}}\right),\left(\mathbf{H}_{\mathbf{3}}\right)$, then the point of convergence given by the SPPM algorithm $\bar{x}$, given by (5) and (6), is a Pareto critical point of the problem (4), that is, there exists $i_{0} \in\{1,2, \ldots, m\}$ such that

$$
\left\langle\nabla F_{i_{0}}(\bar{x}), d\right\rangle \geq 0, \forall d \in \mathbb{R}^{n} .
$$

Proof. It is immediate since continuously differentiable on $\mathbb{R}^{n}$ implies the assumption $\left(\mathbf{H}_{\mathbf{1}}\right)$, and $=F_{i}^{0}(x, d)=F_{i}^{\prime}(x, d)=\left\langle\nabla F_{i}(x), d\right\rangle$, where $F_{i}^{\prime}$ is the directional derivative of $F_{i}$. 


\section{Optimization models with quasiconvex multivalued functions}

In this section we present some general quasiconvex multiobjective problems where the proposed algorithm may be applied.

\subsection{A quasiconvex model in demand theory}

Let $\mathbf{n}$ be a finite number of consumer goods. A consumer is an agent who must choose how much to consume of each good. An ordered set of numbers representing the amounts consumed of each good set is called vector of consumption, and denoted by $x=\left(x_{1}, x_{2}, \ldots, x_{n}\right)$ where $x_{i}$ with $i=1,2, \ldots, n$, is the quantity consumed of good $\mathbf{i}$. Denote by $X$, the feasible set of these vectors which will be called the set of consumption, usually in economic applications we have $X \subset \mathbb{R}_{+}^{n}$.

In the classical approach of demand theory, the analysis of consumer behavior starts specifying a preference relation over the set $X$, denoted by $\succeq$. The notation: " $x \succeq y$ " means that " $x$ is at least as good as $y$ " or " $y$ is not preferred to $x "$. This preference relation $\succeq$ is assumed rational, i.e, is complete because the consumer is able to order all possible combinations of goods, and transitive, because consumer preferences are consistent, which means if the consumer prefers $\bar{x}$ to $\bar{y}$ and $\bar{y}$ to $\bar{z}$, then he prefers $\bar{x}$ to $\bar{z}$ (see Definition 3.B.1 of Mas-Colell et al. [21]).

A function $\mu: X \longrightarrow \mathbb{R}$ is said to be an utility function representing a preference relation $\succeq$ on $X$, if the following condition is satisfied:

$$
x \succeq y \text {, if and only if, } \mu(x) \geq \mu(y)
$$

for all $x, y \in X$.

The utility function is a way to represent preferences between two vectors of consumption. If they have the same value of the utility function, then the consumer is indifferent. Moreover, if we have several preferences relations $\succeq_{i}, i=1,2, \ldots, m$, (multiple criteria), which satisfy the condition (21), then we have a utility function $\mu_{i}$ for each one of these preferences $\succeq_{i}$.

Observe that the utility function not always exist. In fact, define in $X=\mathbb{R}^{2}$ a lexicographic relation, given by: for $x, y \in \mathbb{R}^{2}, x \succeq y$ if and only if " $x_{1}>y_{1} "$ or " $x_{1}=y_{1}$ e $x_{2} \geq y_{2} "$. Fortunately, a very general class of preference relations can be represented by utility functions, see for example 3.C.1 Proposition of Mas-Colell et al. 21.

If a preference relation $\succeq$ is represented by a utility function $\mu$, then the problem of maximizer the consumer preference on $X$ is equivalent to solve the optimization problem

$$
\text { (P) } \max \{\mu(x): x \in X\} \text {. }
$$

Now consider a multiple criteria, that is, consider $m$ preference relations denoted by $\succeq_{i}$ $, i=1,2, \ldots, m$. Suppose that for each preference $\succeq_{i}$, there exists an utility function, $\mu_{i}$, respectively, then the problem of maximizer the consumer preference on $X$ is equivalent to solve the multiobjective optimization problem

$$
\left(\mathrm{P}^{\prime}\right) \max \left\{\left(\mu_{1}(x), \mu_{2}(x), \ldots, \mu_{m}(x)\right) \in \mathbb{R}^{m}: x \in X\right\}
$$


Since there is not a single point which maximize all the functions simultaneously the concept of optimality is established in terms of Pareto optimality or efficiency.

On the other hand, a natural psychological assumption in economy is that the consumer tends to diversify his consumption among all goods, that is, the preference $\succeq$ satisfies the following convexity property: $X$ is convex and if $x \succeq z$ and $y \succeq z$ then $\lambda x+(1-\lambda) y \succeq z$, $\forall \lambda \in[0,1]$.

It can be proved that if there is a utility function representing the preference relation $\succeq$, then the convexity property of $\succeq$ is equivalent to the quasiconcavity of the utility function $\mu$. Therefore $\left(\mathrm{P}^{\prime}\right)$ becomes a maximization problem with quasiconcave multiobjective function, since each component function is quasiconcave.

Taking $\mathrm{F}=\left(-\mu_{1},-\mu_{2}, \ldots,-\mu_{m}\right)$, we obtain a minimization problem with quasiconvex multiobjective function, since each component function is quasiconvex one.

There are various class of utilities functions which are frequently used to generate demand functions. One of the most common is the Cobb-Douglas utility function, which is defined on $\mathbb{R}^{2}$ by $\mu\left(x_{1}, x_{2}\right)=k x_{1}^{\alpha} x_{2}^{\beta}$, with $\alpha, \beta>0$ and $k>0$. Another utility function CES (Constant Elasticity of Substitution), defined on $\mathbb{R}^{2}$ by $\mu\left(x_{1}, x_{2}\right)=\left(\lambda_{1} x_{1}^{\rho}+\lambda_{2} x_{2}^{\rho}\right)^{1 / \rho}$, where $\lambda_{1}, \lambda_{2} \geq 0$, $\lambda_{1}+\lambda_{2}=1$, and $\rho$ is a constant.

\subsection{A quasiconvex model in location theory}

Location problems are related to determining the location for one or more facilities, considering a given set of demand points, with which interactions should be established. These terms are not part of a standard terminology, are sometimes replaced by: clients, existing facilities, businesses or users.

The following problem of locating a facility is motivated from the Chapter IV of Gromicho, [12]. For each $i=1, \ldots, m$, let the cluster set $d^{i}=\left\{d_{1}^{i}, d_{2}^{i}, \ldots, d_{p(i)}^{i}\right\} \subset \mathbb{R}^{n}, n \geq 2$ (there exist $m$ cluster). We need to find a location $x \in \mathbb{R}^{n}$ for an installation so that this location minimizes some real function involving the distance between the new location and each cluster set of demand points.

For each $i=1, \ldots, m$, if $C_{j}^{i}, j=1, \ldots, p(i)$, are compact convex sets with $0 \in \operatorname{int}\left(C_{j}^{i}\right)$ and $\operatorname{int}\left(C_{j}^{i}\right)$ denotes the interior of $C_{j}^{i}$ then, for each $i=1, \ldots, m$, we define the distance between $x$ and $d_{j}^{i}$ by $\gamma_{C_{j}^{i}}\left(x-d_{j}^{i}\right)$ with $\gamma_{C_{j}^{i}}$ the gauge or Minkowsky functional of the $\operatorname{set} C_{j}^{i}$, i.e. $\gamma_{C_{j}^{i}}(x)=\inf \left\{t>0: x \in t C_{j}^{i}\right\}$. Note that if $C_{j}^{i}$ is the unit ball in $\mathbb{R}^{n}$, then $\gamma_{C_{j}^{i}}(x)$ is the Euclidean distance from $x$ to 0 .

To introduce the model, consider, for each $i=1, \ldots, m$, the function $\gamma_{i}: \mathbb{R}^{n} \longrightarrow \mathbb{R}_{+}^{p}$, given by $\gamma_{i}(x)=\left(\gamma_{C_{1}^{i}}\left(x-d_{1}^{i}\right), \ldots, \gamma_{C_{p(i)}^{i}}\left(x-d_{p(i)}^{i}\right)\right)$. And suppose, for each $i$, that the functions $f_{j}^{i}: \mathbb{R}_{+}^{p(i)} \longrightarrow \mathbb{R}_{+}$, with $j=1, \ldots, p(i)$ is nondecreasing in $\mathbb{R}_{+}^{p(i)}$, that is, if $x, y \in \mathbb{R}_{+}^{p(i)}$, satisfying for each $j=1, \ldots, p(i), x_{j} \leq y_{j}$, then $f_{j}^{i}(x) \leq f_{j}^{i}(y)$.

The localization model is given by

$$
\min \left\{\left(\phi_{1}(x), \phi_{2}(x), \ldots, \phi_{m}(x)\right): x \in \mathbb{R}^{n}\right\}
$$

where, for each $i=1, \ldots, m, \phi_{i}(x)=\max _{1 \leq j \leq p(i)} f_{j}^{i}\left(\gamma_{i}(x)\right)$. If for each $i=1, \ldots, m$, the functions $f_{j}^{i}: \mathbb{R}_{+}^{p(i)} \longrightarrow \mathbb{R}^{+}$are quasiconvex in $\mathbb{R}_{+}^{p(i)}$, then it can proved that for every $i=1, \ldots, m$, each function $\phi_{i}($.$) is quasiconvex in \mathbb{R}^{n}$. 


\section{Conclusion and future works}

In this paper we introduced a scalarization proximal point method to solve unconstrained (possibly nonconvex and non-differentiable) multiobjective minimization problems with locally Lipschitz functions. Then, for quasiconvex objective functions we show a strong convergence (global convergence) to a Pareto-Clarke critical point satisfying the completeness assumption $\left(\mathbf{H}_{3}\right)$. Note this assumption has been considered in the convergence analysis of the proximal point method for the convex case, see [3].

We also present, in Section 4, two optimization models where the quasiconvexity of the multiobjective functions appear naturally. We present quasiconvex models in demand theory and location theory.

The (SPPM) algorithm, introduced in this paper, is the first attempt to construct efficient proximal point methods to solve quasiconvex multiobjective minimization problems and in its actual version may be considered as a based algorithm to develop other methods that consider computational errors, lower computational costs, lower complexity order and improves the convergence rate. Observe that in this paper we do not present an inexact version because, according to our knowledge, the theory of $\epsilon$ - subdifferencial Clarke has not yet been developed.

To reduce considerably the computational cost in each iteration of the (SPPM) algorithm it is need to consider the unconstrained iteration

$$
0 \in \partial^{o}\left(\left\langle F(.), z_{k}\right\rangle+\frac{\alpha_{k}}{2}\left\langle e_{k}, z_{k}\right\rangle\left\|\cdot-x^{k}\right\|^{2}\right)\left(x^{k+1}\right)
$$

which is more practical that (6). One natural condition to obtain (22) is that $x^{k+1} \in\left(\Omega_{k}\right)^{0}$ (interior of $\Omega_{k}$ ). So we believe that a variant of the (SPPM) algorithm may be an interior variable metric proximal point method.

Observe also that in practice the iteration (6) or (22) should be solve using a local algorithm, which only provides an approximate solution. Therefore, we consider that in a future work it is important to analyze the convergence of the proposed algorithm considering now inexact iterations, see [28. Also the introduction of bundle methods are welcome.

\section{Acknowledgements}

The research of H.C.F.Apolinário was partially supported by CAPES/Brazil. The research of P.R.Oliveira was partially supported by CNPQ/Brazil. The research of E.A.Papa Quiroz was partially supported by the Postdoctoral Scholarship CAPES-FAPERJ Edital PAPD-2011.

\section{References}

[1] Aussel, D.: Subdifferential properties of quasiconvex and pseudoconvex functions: unified approach. J. Optim Theory Appl. 97, 1, 29-45, (1998).

[2] Bazaraa, M.S., Sherali, H.D. and Shetty, C.M.: Nonlinear Programming:Theory and Algorithms. 3 ed.,John Wiley and Sons, Inc., New York, (2006).

[3] Bello Cruz, J.Y., Lucambio Pérez, L.R. and Melo, J.G.: Convergence of the projected gradient method for quasiconvex multiobjective optimization. Nonlinear Analysis. 74, 5268-5273 (2011). 
[4] Bolte, J., Daniilidis, A.,Lewis, A. and Shiota,M.: Clarke subgradients of stratifiable functions. SIAM Journal on Optimization. 18,556-572 (2007).

[5] Bonnel, H., Iusem, A.N. and Svaiter, B.F.: Proximal methods in vector optimization. SIAM Journal on Optimization. 15, 953-970 (2005).

[6] Brito, A.S, da Cruz Neto, J.X, Lopes, J.O, Oliveira, P.R: Interior proximal algorithm for quasiconvex programming and variational inequalities with linear constraints. J. Optim Theory Appl. 154, 217-234, (2012).

[7] Ceng, L. and Yao, J.: Approximate proximal methods in vector optimization. European Journal of Operational Research. 183, 1-19(2007).

[8] Clarke, H.F.: Optimization and nonsmooth analysis. Classics in applied mathematic, SIAM, New York (1983).

[9] Custodio, A.L., Madeira, J.F.A., Vaz, A.I.F and Vicente, L.N.: Direct Multisearch for multiobjective optimization. SIAM Journal on Optimization. 21, 1109-1140 (2011).

[10] Da Cruz Neto, J.X., Da Silva, G.J.P., Ferreira, O.P., Lopes, J.O.: A subgradient method for multiobjective optimization. Computational Optimization and Applications. 54 (3), 461-472,(2013).

[11] Gregório, R. and Oliveira, P.R. : A Logarithmic-quadratic proximal point scalarization method for multiobjective programming. Journal of Global Optimization. 49, 361-378 (2010).

[12] Gromicho J.: Quasiconvex optimization and location theory. Kluwer Academic Publishers, Dordrecht, the Netherlands, (1998)

[13] Güler, O.: New proximal point proximal algorithms for convex minimization. SIAM Journal Control and Optimization. 2, 649-664 (1992).

[14] Hadjisavvas, N., Komlosi, S. and Shaible, S.: Handbook of Generalized Convexity and Generalized Monotonicity. Nonconvex Optimization and its Applications 76, SpringerVerlag, New York, (2005).

[15] Huang, X.X. and Yang, X.Q.: Duality for multiobjective optimization via nonlinear Lagrangian functions. Journal of Optimization Theory and Applications. 120, 111$12(2004)$.

[16] Kiwiel, K.C.: Convergence and efficience of subgradient methods for quasiconvex minimization. Math Program. A 90, 1-25 (2001)

[17] Langenberg, N. and Tichatschke, R.: Interior proximal methods for quasiconvex optimization. J Glob Optim. 52, 641-661 (2012).

[18] Luc, T.D.: Theory of vector optimization, Lecture Notes in Economics and Mathematical Systems. Springer, Berlin, (1989). 
[19] Mangasarian, O.L.: Nonlinear Programming. McGraw-Hill, New York, (1969).

[20] Martinet, B.: Regularization d'inequations variationelles par approximations sucessives. Révue Française d'informatique et Recherche Opérationelle. 4, 54-159 (1970).

[21] Mas-Colell, A. Whinston, M.D., Green, J.R.: Microeconomic theory. Oxford University Press, New York, NY, USA, (1995).

[22] Miettinen, K.M.: Nonlinear multiobjective optimization. Kluwer Academic Publishers, Boston, (1999).

[23] Mordukhovich, B.S.: Variational analysis and generalized differentiation I: Basic theory. Grundlehren Series[Fundamental Principles of Mathematical Sciences], vol. 330, SpringerVerlag, Berlin, (2006).

[24] Papa Quiroz, E.A., Oliveira, P.R.: Proximal point methods for quasiconvex and convex functions with Bregman distances on Hadamard manifolds. Journal of Convex Analysis. 16, 1, 49-69 (2009).

[25] Papa Quiroz, E.A., Oliveira, P.R.: Full Convergence of the proximal point method for quasiconvex functions on Hadamard manifolds. ESAIM: COCV, 18, 483-500, (2011).

[26] Papa Quiroz, E.A. and Oliveira, P.R.: An extension of proximal methods for quasiconvex minimization on the nonnegative orthant. European Journal of Operational Research. 216, 26-32 (2012).

[27] Papa Quiroz, E.A. and Oliveira, P.R.: Proximal point method for minimizing quasiconvex locally Lipschitz functions on Hadamard manifolds. Nonlinnear Analysis. 75, 5924-5932, (2012).

[28] Papa Quiroz, E.A., Mallma Ramirez L., and Oliveira, P.R. An inexact proximal method for quasiconvex minimization, submitted paper, 2013.

[29] Rockafellar, R.T. and Wets, R.J-B.: Variational Analysis. Springer, Berlin, (1998).

[30] Schott, D.: Basic properties of Fejer monotone sequences. Rostocker Mathematische Kolloquium. 49, 57-74 (1995).

[31] Villacorta, K.D.V. and Oliveira, P.R.: An interior proximal method in vector optimization. European Journal of Operational Research. 214, 485-492 (2011). 